Hydroécol. Appl. (1992) Tome 4 Vol 2, pp. 159-172

\title{
Les rejets urbains en mer
}

\author{
J.F. Guillaud \\ IFREMER Centre de Brest, DRO/EL B.P. 70, 29280 Plouzane - France
}

Résumé. - Les principaux résultats du programme d'étude de l'IFREMER sur les rejets urbains en mer confirment tout d'abord quels sont les taux d'élimination des contaminants majeurs au cours de leur traitement dans les stations d'épuration biologique de Morlaix et physico-chimique de Toulon. La dilution des effluents dans la zone côtière est ensuite évaluée à l'aide de modèles mathématiques d'advection-dispersion ef le devenir des sels nutritifs, des micropolluants organiques et minéraux, et des microorganismes est décrit. Enfin, les effets des contaminants urbains sont évalués en terme d'eutrophisation pour les sels nutritifs, de toxicité sublétale pour les micropolluants et de contamination microbienne dans les zones de baignade et de conchyliculture. En conclusion, des améliorations concernant le traitement et le rejet en mer des effluents urbains sont proposées.

Mots-clés. - Effluents urbains, zone côtière, sels nutritifs, micropolluants, microorganismes.

Abstract. - The main results of IFREMER study program on the urban waste water discharges into the sea give firstly the elimination rates of contaminants through biological and physical-chemical sewage treatment plants. Then the waste water dilution in the coastal zone is predicted by mathematical modeling and the fate of nutrients, mineral and organic micropollutants, and microorganisms is described. Finally the effects of urban contaminants are evaluated in terms of eutrophication for nutrients, sublethal effects for micropolluants and sanitary problems for microorganisms in bathing and shellfishing areas. In conclusion, main improvements of waste water treatment and disposal in the coastal zone are proposed.

Key-words. - Waste water, coastal zone, nutrients, micropollutants, microorganisms.

\section{INTRODUCTION}

La prise de conscience de l'importance des rejets urbains vis-à-vis du milieu marin est récente et ce, malgré les impacts directs de ces rejets sur l'environnement littoral et sur les nombreuses activités côtières (risques d'eutrophisation et de contamination microbiologique ou chimique); c'est 
en fait depuis les années 70 , et à l'aide des connaissances scientifiques alors disponibles, que des mesures réglementaires et des moyens techniques ont été mis en place pour limiter au mieux l'impact de ces rejets en mer. (Ministère de l'Environnement, 1983).

Afin de progresser dans la résolution des problèmes que posent les rejets urbains en zone côtière, l'IFREMER a, depuis 1986, développé sur ce thème, une recherche pluridisciplinaire originale. L'objectif essentiel de ce programme d'étude est de déterminer le devenir et les effets des effluents urbains déversés en mer, à partir d'une meilleure prise en compte des processus et des mécanismes spécifiques au milieu littoral (Guillaud, 1987).

Ces travaux ont été entrepris en collaboration avec de nombreux laboratoires de recherche français et avec le concours de l'Agence de l'Eau Rhône-Méditerranée-Corse.

\section{MÉTHODES ET SITES D'ÉTUDES}

Pour des raisons d'efficacité, la majorité des études pluridisciplinaires, nécessaires au déroulement de ce programme, a été volontairement focalisée sur deux sites littoraux, représentatifs des technologies d'épuration utilisées et des configurations de rejets rencontrés en zone côtière.

Le premier site de rejet est celui de Morlaix sur le littoral de la Manche, où les effluents épurés se déversent en tête d'un estuaire soumis à une marée de grande amplitude $(9 \mathrm{~m}$ en vive-eau) et qui comporte de larges estrans occupés, dans la partie aval, par des parcs ostréicoles (fig. 1). La station d'épuration réalise un traitement biologique à boue activée forte charge des effluents issus de l'agglomération (24000 habitants).

Le deuxième site est celui de TouIon-Est sur le littoral méditerranéen; la station d'épuration collecte les effluents d'environ 65000 habitants et les eaux épurées sont rejetées à 43 mètres de profondeur grâce à un émissaire de 1800 mètres de long (fig. 2). La station physico-chimique de Toulon-Est met en œuvre la floculation par précipitation de l'hydroxyde ferrique obtenu par addition de chlorosulfate ferrique, de chaux et de polymères organiques. Les boues sont récupérées par décantation lamellaire. La zone de rejet, située dans la grande Rade de Toulon, est caractérisée par des courants relativement faibles, en particulier en dessous de 10 mètres de profondeur, et par l'installation d'une thermocline en période estivale.

Le programme d'étude a tout d'abord consisté à déterminer les flux de contaminants dans les effluents bruts et dans les effluents épurés des deux stations. Ces mesures ont été réalisées lors de campagnes ponctuelles avec plusieurs prélèvements durant 24 heures, et lors des campagnes de longue durée (6 mois à un an) avec prélèvement chaque semaine d'un échantillon quotidien proportionnel au 


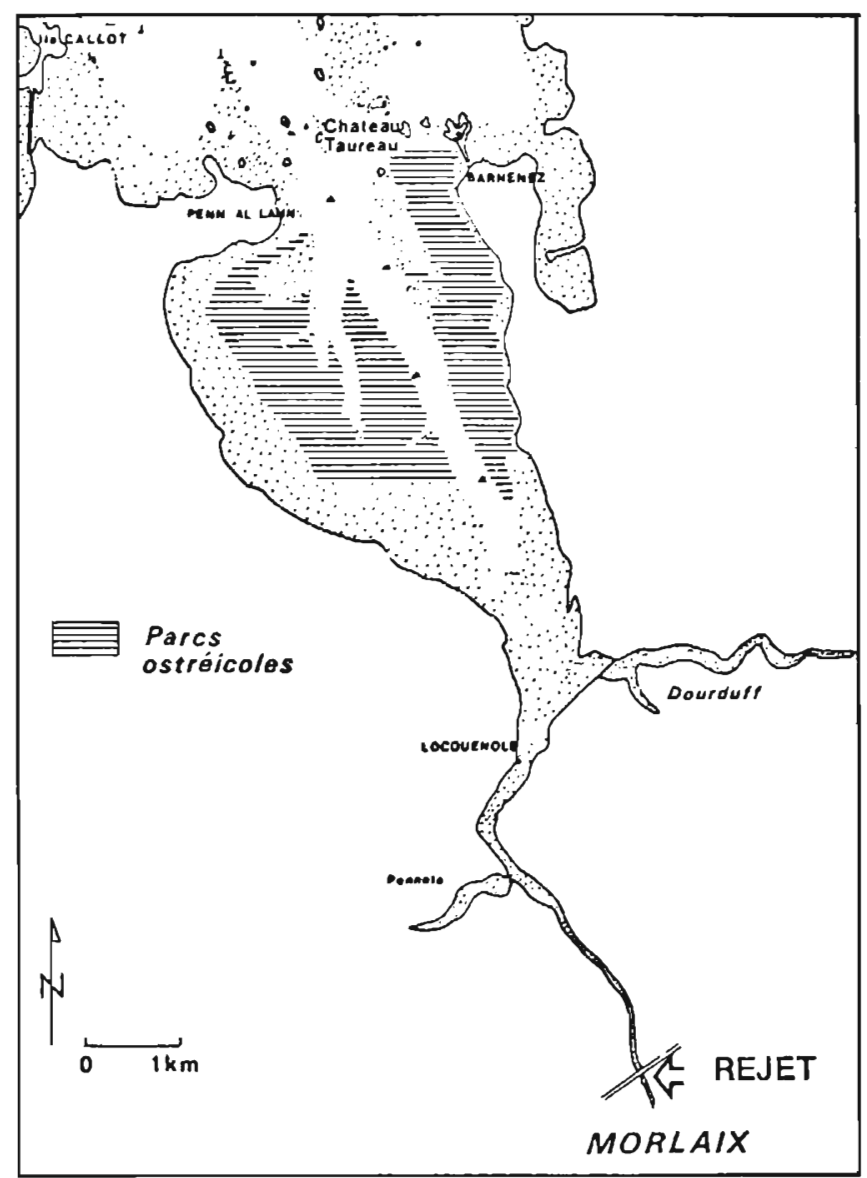

Fig. 1. - Le site d'étude de Morlaix

Fig. 1. - Study site of Morlaix

débit. Les analyses ont porté sur les paramètres classiques de pollution (MES, $\mathrm{DBO}_{5}, \mathrm{DCO}$ ) et sur la quantification d'un grand nombre de contaminants comprenant:

- Les différentes formes de matières organiques, de l'azote et du phosphore;
- les bactéries (coliformes fécaux, streptocoques fécaux, salmonelles) et les virus (enterovirus, VHA),

- les micropolluants organiques (hydrocarbures, PCB, Lindane, solvants chlorés, chlorophénols, détergents);

- les métaux ( $\mathrm{Cu}, \mathrm{Zn}, \mathrm{Pb}, \mathrm{Cd} . .$.$) .$ 


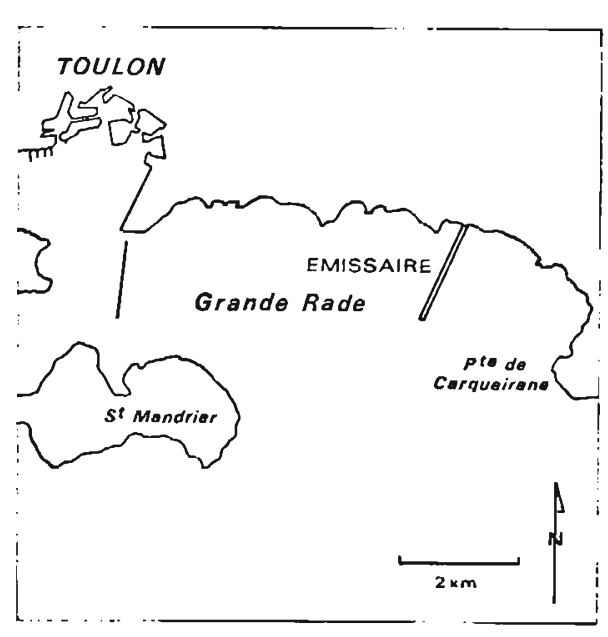

Fig. 2. - Le site d'étude de Toulon

Fig. 2. - Study site of Toulon

Dans un deuxième temps, les processus intervenant au cours de la dispersion des contaminants dans la zone côtière, ont été examinés grâce à différentes campagnes de mesure in situ; à Toulon la dispersion des contaminants dans le champ proche a été suivie à l'aide de traceurs radioactifs ou colorés injectés dans les effluents, en sortie de station; ces mesures ont eu lieu en périodes stratifiées, durant lesquelles l'effluent reste bloqué en profondeur, et en période homogène, où l'effluent remonte en surface. Dans l'estuaire de Morlaix, les campagnes de mesures ont été faites dans différentes conditions de débits (crue-étiage) et de marée (pleine mer, basse mer, vive-eau, morte-eau).

Par ailleurs, des modèles mathématiques d'advection-dispersion ont été mis en place sur les deux sites d'étude. A Toulon, la modélisation a tout d'abord porté sur la remontée du panache et la profondeur de captage en fonction des conditions de stratification thermique et des courants; dans une deuxième phase le modèle calcule, en fonction des courants, le transport et le mélange du nuage de pollution formé par le panache étalé en surface ou en profondeur, en cas de captage sous la thermocline (Thouvenin, 1991).

Sur le site de Morlaix, le transport et la dispersion des effluents urbains sont calculés à l'aide d'un modèle mathematique unidimensionnel dans la partie amont et bidimensionnel horizontal dans la partie aval (Salomon et al., 1991). Cette modélisation de la dispersion des éléments dissous est complétée par un modèle de transport des sédiments cohésifs qui peut être utilisé pour simuler le transport de contaminants particulaires ou adsorbés sur les matières en suspension. (Le Hir et al., 1991).

Enfin, l'étude de l'effet des contaminants a été entreprise à la fois sur l'effluent global après épuration, au moyen de tests portant sur l'altération des processus de reproduction et de développement larvaire d'organismes tels que les oursins ou les huîtres, et sur l'examen de la toxicité létale et sublétale de certains contaminants particuliers. L'effet des rejets de microorganismes par les effluents urbains a été étudié au niveau des zones conchylicoles en examinant les processus d'accumulation des bactéries par les mollusques filtreurs. 


\section{RÉSULTATS}

Les résultats présentés porteront essentiellement sur les apports, le devenir et les effets des sels nutritifs (azote et phosphore), des micropolluants organiques et métalliques, et des microorganismes rejetés en zone littorale par les stations d'épuration. Auparavant on examinera les conditions de dilution des effluents sur les deux sites.

\subsection{La dilution des effluents}

Sur le site de Morlaix, la dilution des effluents est essentiellement fonction des conditions de débit et de marée; le modèle mathématique d'advectiondispersion mis en place permet tout d'abord de déterminer la concentration d'un élément conservatif rejeté par la station en un point quelconque du site et à un instant donné; il permet aussi de cartographier (fig. 3) l'impact «nominal » du rejet d'un élément conservatif, particulaire ou dissous, en calculant en chaque point, en fonction du flux rejeté, les concentrations maximales induites par différentes conditions de marée (Le Hir et al., 1991). Les dilutions les plus faibles de l'effluent, au sud de la baie, sont comprises entre 50 et 100 (basse mer, vive-eau, étiage).
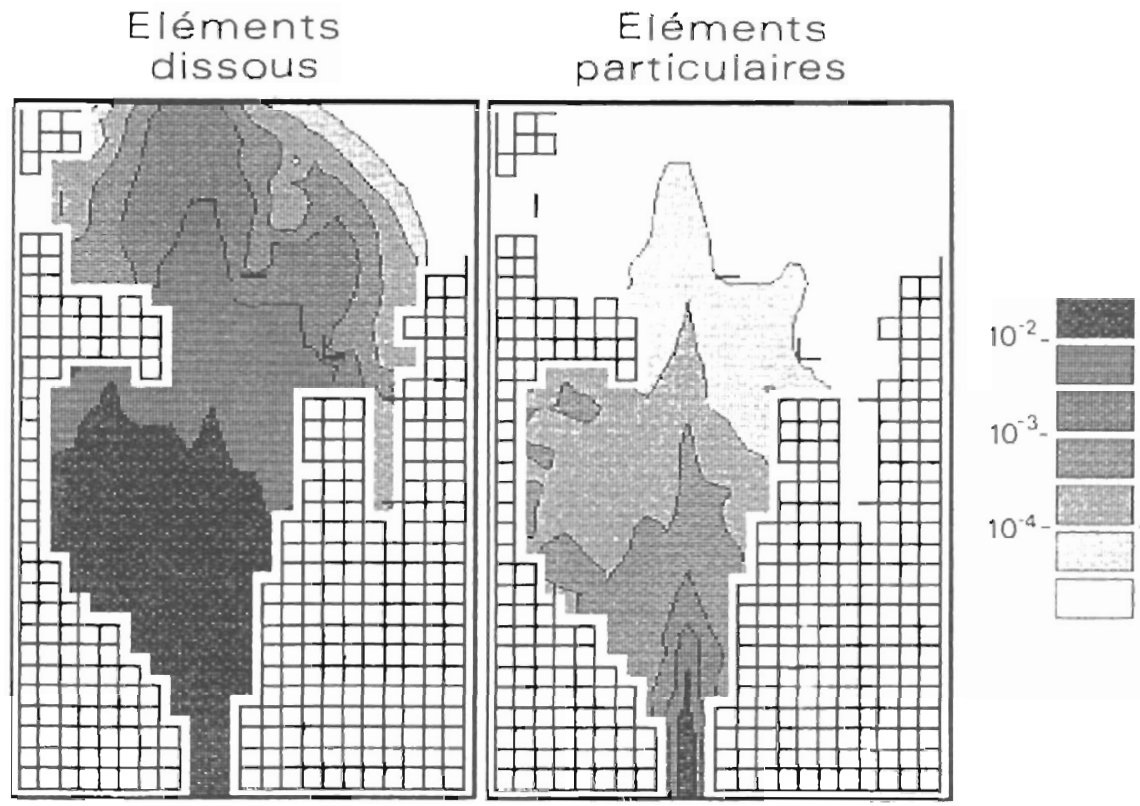

Fig. 3. - Impact "nominal » d'un rejel conservatif à la station d'épuration de Morlaix. Concentrations $\left(\right.$ par $\mathrm{m}^{3}$ ) relatives à un flux unité $\left(=1 ; \mathrm{s}^{-1}\right)$. (in Le Hir et al., 1991).

Fig. 3. - "Nominal" impact of a conservative waste discharge from Morlaix sewage treatment plan. Concentrations $\left(\right.$ per $\left.\mathrm{m}^{3}\right)$ for an unitary flux $\left(1 . \mathrm{s}^{-1}\right)$. (in Le Hir et al., 199). 
Sur le site de Toulon, la dilution des effluents a lieu tout d'abord durant la remontée dans le panache, puis lors du transport horizontal du nuage de pollution. Un premier modèle mathématique permet de calculer la dilution des effluents lors de leur remontée, et la profondeur du blocage éventuel sous la thermocline, en fonction du débit du rejet, de la bathymétrie, des courants et de la stratification du milieu; un deuxième modèle mathématique de transport et de mélange calcule la dispersion horizontale de l'effluent dans le champ proche en fonction des courants et de leur variabilité sur la profondeur (Thouvenin, 1991).

Lors de la remontée des effluents dans le panache, les ordres de grandeur de la dilution sont, pour un débit moyen de $600 \mathrm{~m}^{3} / \mathrm{h}$ et une profondeur de $40 \mathrm{~m}$, de 50 à 150 en surface et de 40 à 60 si l'effluent est bloqué à $20 \mathrm{~m}$ de profondeur sous la thermocline.

Lors de la deuxième étape de dilution (transport horizontal) il existe une zone de mélange intense (diffusion importante, écoulement radial) de 100 à $200 \mathrm{~m}$ de diamètre où la dilution est multipliée, par un facteur compris entre 2 et 20 en surface et entre 1,5 et 4 en profondeur. Au delà de $200 \mathrm{~m}$, la dilution n'est multipliée que par un facteur 1,5 à 3 tous les 500 mètres.

\subsection{Les sels nutritifs}

Les apports en éléments nutritifs par les stations d'épuration dépendent non seulement des flux d'effluents bruts reçus, mais aussi des rende- ments d'élimination de l'azote et du phosphore au cours du transit dans les installations d'épuration.

Dans les stations d'épuration biologiques, sans procédé spécifique de dénitrification, les rendements d'élimination de l'azote total oscillent de 30 à $80 \%$ selon qu'il s'agit de station à forte ou à faible charge; à Morlaix, station à forte charge, ces rendements sont actuellement de $32 \%$ (Aminot et al., 1990). L'élimination du phosphore total dans les stations biologiques à boues activées se fait avec un rendement plus faible généralement compris entre 20 et $50 \%$ (Schaack et al., 1985); à Morlaix on observe actuellement un rendement de $26 \%$ (Aminot et al., 1990).

Dans les stations physico-chimiques la situation est inverse avec un rendement plus faible, compris entre 5 et $20 \%$, pour l'élimination de l'azote total ( $6 \%$ à la station de Toulon-Est), et une élimination plus forte du phosphore, généralement supérieure à $60 \%(67 \%$ à Toulon-Est) du fait de leur principe de fonctionnement fondé sur la floculation chimique (Aminot et al., 1991).

L'ensemble des apports en sels nutritifs, qui sont des éléments nécessaires à la croissance des végétaux en zone littorale, peuvent devenir des facteurs d'eutrophisation sur certains sites sensibles du fait de leurs caractéristiques morphologiques ou hydrodynamiques. Lorsqu'il y a apport excessif de sels nutritifs sur une zone littorale, il convient, avant d'engager des investissements pour réduire les rejets urbains, de bien cerner les parts 
relatives dues aux effluents urbains et aux fleuves côtiers. Dans certaines régions agricoles, comme la Bretagne, on constate souvent que les flux d'azote en provenance des bassins versants sont bien supérieurs à ceux issus des agglomérations littorales; la situation est cependant moins tranchée pour le phosphore (Aminot, 1991). Sur le littoral méditerranéen, l'existence d'upwellings côtiers, par exemple en régime de mistral, peut constituer une source importante d'eau de fond, plus riche en sels nutritifs.

Le devenir des sels nutritifs apportés à la zone littorale sera essentiellement fonction des processus de dilution physique, de consommation par les végétaux et, éventuellement, d'adsorption-désorption sur le matériel particulaire. Dans les zones méditerranéennes ouvertes, où les rejets par émissaire se font en profondeur, on observe comme sur le site de Toulon, une première dilution rapide des sels nutritifs, par un facteur égal ou supérieur à 100, lors de la remontée des effluents en surface; ensuite lors de la dispersion horizontale dans le champ proche, les concentrations en azote et en phosphore atteignent, à une distance de l'émissaire comprise entre 800 et $1000 \mathrm{~m}$, la gamme de variation naturelle des teneurs en sels nutritifs des eaux côtières. Contrairement aux risques de dystrophie que représentent les rejets de sels nutritifs par les apports urbains dans les étangs méditerranéens, il apparaît que les apports d'éléments nutritifs d'origine urbaine dans des zones ou- vertes et oligotrophes, telles que la Rade de Toulon, ne semblent pas susceptibles d'entraîner de grave problème d'eutrophisation. Ceci est confirmé par l'absence d'augmentation des teneurs en chlorophylle dans le champ proche de l'émissaire et par l'absence de déficit en oxygène dissous au niveau du fond ( $A$. Romana, comm. pers.).

Dans les zones estuariennes, comme à Morlaix, les apports d'azote par les rejets urbains sont souvent faibles par rapport aux flux de nitrate issus des bassins versants notamment en région agricole; par contre, les rejets urbains d'ammonium (qui constitue souvent la forme prépondérante de l'azote rejeté par les stations d'épuration) peuvent provoquer une augmentation significative des concentrations en cet élément dans le milieu; cette forme réduite de l'azote minéral, susceptible d'entrainer une consommation d'oxygène dissous dans l'estuaire lors de son oxydation, peut aussi se révéler toxique, notamment pour les salmonidés, par sa forme non ionisée $\left(\mathrm{NH}_{3}\right)$; cette dernière est en équilibre avec l'ion ammonium et cet équilibre est régi par les conditions de $\mathrm{pH}$, de température et de salinité; la valeur impérative proposée par la C.E.E. pour le maintien de la qualité des eaux salmonicoles, qui est de $0,025 \mathrm{mg} / \mathrm{l}$ de $\mathrm{NH}_{3}$ (Martin, 1979), est parfois dépassée sur le site de Morlaix.

Les apports de phosphate en estuaire par les stations d'épuration représentent en général une proportion plus importante que les apports ur- 
bains d'azote, par rapport aux flux issus du bassin versant. Le phosphate rejeté sera capable de s'adsorber sur les sédiments qui, lors des remises en suspension par les courants estuariens, passent en phase oxique (fig. 4); le phosphore ainsi stocké dans les sédiments superficiels fins pourra être relargué lorsque ces sédiments redeviennent anoxiques et lorsque les concentrations dans l'eau surnageante sont suffisamment basses (Guillaud et Aminot, 1991).

Les risques d'eutrophisation en zone Manche-Atlantique seront fonction non seulement des apports de nutriments, mais aussi du confinement géographique ou hydrodynamique (faible circulation résiduelle de marée) des zones littorales où s'effectuent les rejets urbains ou agricoles (Menesguen, 1991). Dans ces sites sensibles à l'eutrophisation, qui connaissent des développements im-

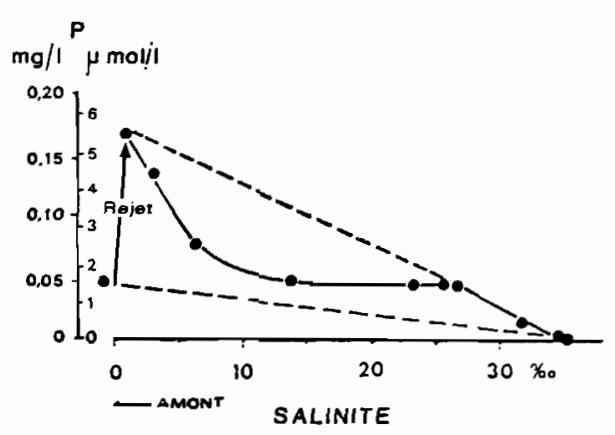

Fig. 4. - Concentrations en phosphate dans l'estuaire de Morlaix en période de forte remise en suspension.

Fig. 4. - Phosphate concentrations in Morlaix estuary with high suspended matter concentrations. portantes de macro-algues ou de phytoplancton, il convient tout d'abord de bien relativiser les apports urbains par rapport aux flux d'éléments nutritifs en provenance des bassins versants; cette comparaison doit être faite notamment en périodes critiques printanière et estivale, et elle peut permettre de fixer des priorités dans la lutte contre les apports excessifs de nutriments.

Dans le cas d'apports urbains prépondérants dans une zone à risque d'eutrophisation, il convient alors d'identifier si un sel nutritif (azote ou phosphore) est plus particulièrement limitant pour la production primaire et si donc l'effort de lutte peut être mieux ciblé dans la filière d'épuration. Cette identification peut s'effectuer par la comparaison entre des cultures phytoplanctoniques en milieux diversement enrichis en éléments nutritifs, et les cultures en milieu témoin (Ryther et Dunstan 1971; Queguiner 1988). Une autre méthode consiste à suivre, pour les macro-algues, le contenu des tissus végétaux en azote et en phosphore, de façon à déterminer l'élément pour lequel l'algue est la plus carencée en période productive (Dion, 1988).

En zone littorale Manche-Atlantique l'élément limitant est en général l'azote, sauf dans certains secteurs côtiers fortement influencés par l'arrivée d'un fleuve, comme la Baie de Vilaine (Queguiner, 1988); les rapports molaires N/P sont du reste généralement inférieurs dans le milieu marin par rapport aux rivières où le phosphore est souvent limitant. Par ailleurs, les 
sédiments fins littoraux seraient une source plus importante de phosphore que d'azote, pour la colonne d'eau, en cas de forte consommation par la biomasse végétale (Lerat, 1990).

\subsection{Les micropolluants organiques et métalliques}

Les taux d'élimination des micropolluants organiques dans la station à boues activées de Morlaix sont, en régime normal, supérieurs à $80 \%$, à l'exception de composés tels que le lindane ou le pentachlorophenol, (taux d'abattement compris entre 10 et $20 \%$ ) qui sont peu dégradables, peu adsorbables, faiblement volatils et donc mal éliminés. Dans la station physico-chimique de Toulon, les taux d'élimination de la majorité des micropolluants organiques sont plus faibles et compris entre 40 et $70 \%$ (Romana et al., 1991).

Les rendements d'élimination des métaux varient de 60 à $80 \%$ dans les deux types de station; au cours du suivi à long terme de la station de Morlaix, une relation entre l'élimination des métaux et l'élimination des matières en suspension (MES) a été mise en évidence; elle est, par exemple pour le cuivre et le plomb, de la forme:

$$
\begin{array}{r}
\text { rdt Cu }(\%)=0,79 \operatorname{rdtMES}(\%)+2,66 \\
n=22 ; r=0,80
\end{array}
$$

rdt $\mathrm{Pb}(\%)=0,67$ rdtMES $(\%)+10,64$

$$
n=22 ; r=0,70
$$

Les apports moyens journaliers par habitant au sortir des deux stations d'épuration sont reportés dans le tableau I (Marchand et al., 1989; Romana et al., 1991). Les valeurs varient de plusieurs ordres de grandeur en fonction des différents contaminants, mais sont relativement proches, pour un même polluant, dans les deux filières, et donnent une bonne évaluation des apports par habitant pour des agglomérations faiblement industrialisées.

Tableau 1. - Apports quotidiens par habitant en micropolluants par les stations de Morlaix et Toulon.

Table 1. - Per capita daily fluxes of micropollutants from Morlaix and Toulon sewage treatment plants.

\begin{tabular}{|l|cc|} 
& \multicolumn{2}{|c|}{$\mathrm{mg} / \mathrm{j} / \mathrm{hab}}$. \\
& Morlaix : Toulon \\
\hline Détergents anioniques & 300 & 2000 \\
Hydrocarbures totaux & 700 & 340 \\
Hydroxarbures aromatiques & 3 & 4 \\
PCB & 0,01 & 0,03 \\
$\mathrm{Zn}$ & 26 & 18 \\
$\mathrm{Cu}$ & 5 & 4 \\
$\mathrm{~Pb}$ & 2 & 3 \\
$\mathrm{Cd}$ & 0,1 & 0,1 \\
\hline
\end{tabular}

L'étude à long terme des rejets de la station de Toulon a permis de montrer l'existence de périodes préférentielles d'apport pour certains polluants, telles que la fin de la semaine pour les hydrocarbures aromatiques, la saison estivale pour le lindane et le cadmium, ou les épisodes orageux pour les PCB.

Afin d'évaluer la toxicité des effluents rejetés et en particulier des contaminants qu'ils contiennent, un 
recensement des concentrations les plus faibles de polluants induisant un effet sublétal a été fait à partir des données de la littérature ou des résultats d'expériences (Lassus et al., 1991). Ces valeurs ont été comparées aux concentrations les plus fortes pouvant être trouvées dans le milieu, à la sortie des rejets de Toulon et Morlaix (fig. 5). II en ressort que les dilutions instantanées minimales (de l'ordre de $1 / 50^{e}$ ) des effluents dans le milieu suffisent à ramener les teneurs au-dessous des seuils toxiques les plus bas, à l'exception des détergents anioniques qui peuvent constituer un risque pour l'environnement. Les plus faibles rendements d'élimination des détergents constatés à Toulon (40\%) qu'à Morlaix (91\%), conduisent au rejet de 47 Van de détergents anioniques sur ce premier site et de 3 t/an sur le second. Les détergents anioniques rejetés en mer sont aussi une cause indirecte de la dégradation de la végétation littorale par l'intermédiaire des aérosols marins riches en détergents et susceptibles d'attaquer les cires végétales (Giovanelli et al., 1988).

La toxicité globale des effluents traités a aussi été évaluée à l'aide de tests écotoxicologiques. A partir des effluents de Morlaix, I'on a examiné l'action de différentes dilutions de l'effluent dans l'eau de mer, sur le développement embryonnaire de larves de moules et d'huitres; les taux d'anomalies sont significativement supérieurs au témoin pour des dilutions de l'effluent inférieures au $50^{\mathrm{e}}$ (Lassus et al., 1991).

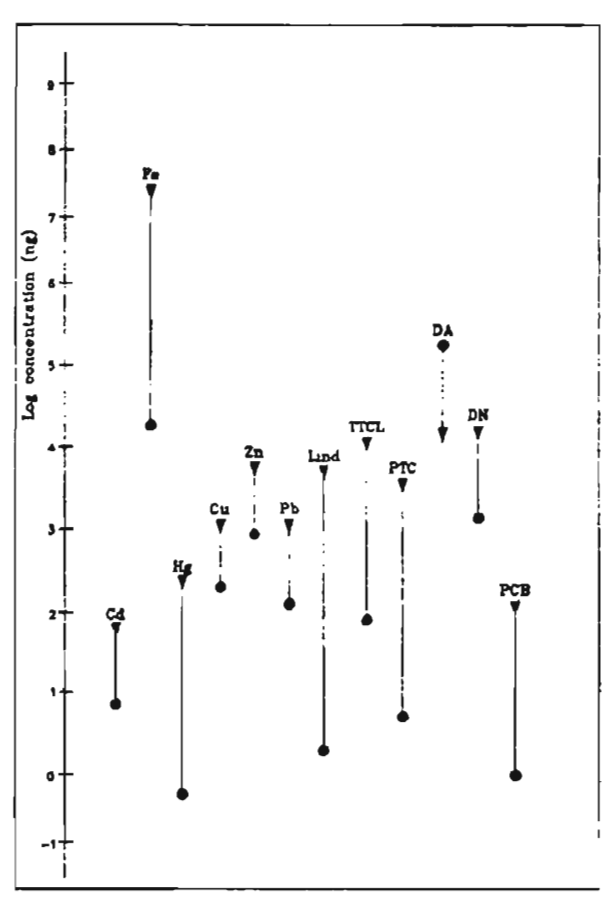

Fig. 5. - Concentrations de contamlinants les plus élevées à la sortie des rejets de Toulon et Morlaix (cercles noirs) et concentrations les plus faibles induisant un effet sublétal (triangle noir). TTCL: tetrachloroéthylene, PTC; pentachlorophénols, D.A. : détergents anioniques, DN: détergents non-ioniques. (in Lassus et al., 1991). Fig. 5. - Highest pollutant concentrations near Toulon and Morlaix waste discharges (black circles) and lowest concentrations inducing sublethal effects (black triangle). TाC: tetrachioroethylene, PTC: pentachlorophenols, D.A. : anionic detergents, DN : non-ionic detergents. (in Lassus et al., 1991).

En ce qui concerne l'effluent épuré de Toulon, des tests concernant la spermiotoxicité et l'embryotoxicité de l'oursin ont été réalisés; ces tests révèlent une toxicité pour des dilutions de l'effluent inférieures à $1 / 250^{\mathrm{e}}$ et cet 
effet toxique semble lié à l'ajout de floculant au cours du traitement physico-chimique, sans que le mécanisme n'ait pu être clairement déterminé; on retrouve cet effet toxique dans les sédiments superficiels autour de l'émissaire, en particulier dans la fraction fine inférieure à $63 \mu \mathrm{m}$.

L'estimation des zones soumises à un effet toxique direct des effluents, sans prendre en compte les processus de bioaccumulation possible, repose donc sur la détermination de l'aire géographique ou la dilution est inférieure à une valeur de l'ordre de $1 / 250^{e}$; l'importance de cette zone est fonction des conditions hydrodynamiques; elle peut atteindre $6 \mathrm{~km}$ dans le cas de Morlaix (étiage, basse-mer de vive-eau) et une dizaine de $\mathrm{km}$ dans le cas de Toulon (faible courant de surface).

\subsection{Les micro-organismes}

Les concentrations élevées en microorganismes dans les effluents urbains bruts (coliformes fécaux de l'ordre de $10^{8} / 1$, particules virales pouvant atteindre $\left.10^{3} / \mathrm{l}\right)$ ne sont réduites en moyenne que d'une unité logarithmique dans les stations biologiques classiques, comme à Morlaix, et de moins de 0,5 unité logarithmique dans les stations physico-chimiques, comme à Toulon. Le taux d'élimination des bactéries fécales, dans les stations biologiques, et par ailleurs lié à celui des matières en suspension (Dupray et al., 1991).

Lorsque les bactéries rejetées arrivent en mer, elles vont être soumises à plusieurs stress qui sont dus, en autre, à l'augmentation de la pression osmotique, à l'oligotrophie et à l'action de la lumière.

Dans les zones riches en matières organiques, il semble que les bactéries trouvent dans l'eau et dans les sédiments des osmoprotecteurs, du type bétaiine, qui leur permettent de développer des mécanismes d'halotolérance (Pommepuy et al., 1991).

Par ailleurs, le temps de disparition des bactéries en mer est lié à l'énergie lumineuse reçue par ces bactéries (fig. 6); cette énergie sera fonction de l'ensoleillement, de la turbidité des eaux et de la profondeur; ainsi on observe des T90 (temps de disparition de $90 \%$ des bactéries) de l'ordre de l'heure, en Méditerranée, lorsque les effluents sont en surface, et de plusieurs centaines d'heures en zone es-

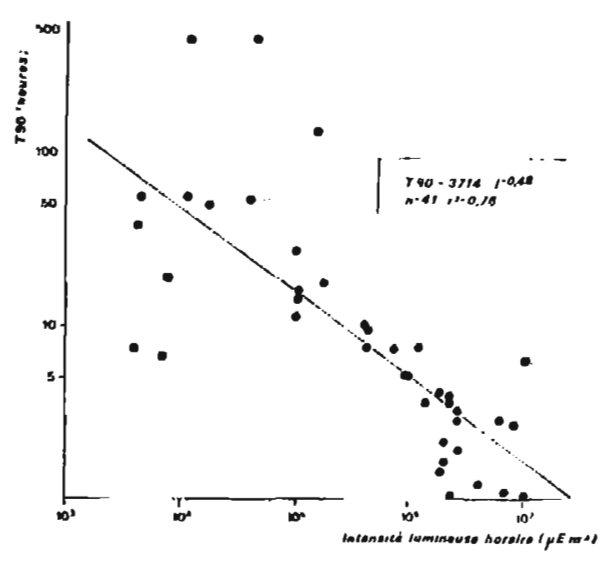

Fig. 6. - Relation entre le T90 et l'intensité lumineuse horaire (in Pommepuy et al., 1991).

Fig. 6. - Relationship between T90 and hourly light intensity (in Pommepuy et al., 1991). 
tuarienne turbide de l'Atlantique; dans ce dernier cas les processus physiques de dilution joueront un plus grand rôle que la mortalité biologique dans la diminution des concentrations en bactéries dans la zone de rejet.

Bien que les bactéries pathogènes constituent un risque pour la santé dans les zones balnéaires, il semble bien que les conséquences épidémiologiques les plus importantes concernent les zones où se pratique la conchyliculture; en effet, les coquillages filtreurs tels que les huitres, sont capables d'accumuler en un temps très bref (de l'ordre de $30 \mathrm{~mm}$ en été et de 5 heures en hiver) les bactéries présentes dans l'eau; les facteurs d'enrichissement par rapport à l'eau varient de 10 pour les coliformes fécaux à plus de 100 pour les streptocoques fécaux (Prieur et al., 1990).

\section{CONCLUSION}

L'amélioration des conditions de traitement et de rejet des effluents urbains en zone littorale passe par plusieurs étapes qui concernent:

- l'optimisation du choix des points de rejets sur chaque site, à l'aide notamment de la modélisation mathématique, permettant d'évaluer, en fonction des caractéristiques hydrodynamiques de la zone, la meilleure dilution possible; celle-ci représente souvent le facteur le plus efficace pour diminuer les concentrations en contaminants biodégradables;
- la recherche et le maintien d'un niveau correct d'élimination des micropolluants rémanents; on peut ici remarquer que les contaminants organiques qui ont un fort taux de bioaccumulation ont aussi un taux élevé d'adsorption sur le matériel organique particulaire car ces deux paramètres sont directement fonction du coefficient de partage octanol/eau (Marchand, 1985); l'élimination poussée des matières en suspension organiques devrait donc en diminuer les apports;

- la réduction des apports en azote et/ou en phosphore, par des procédés aujourd'hui bien maîtrisés en station d'épuration; cette réduction ne devra être mise en place que dans le cas où la zone de rejet est sensible à l'eutrophisation et où les rejets urbains constituent l'essentiel des apports en sels nutritifs; cette action nécessite aussi de définir clairement quel est l'élément nutritif limitant dans la zone eutrophe;

- la diminution des apports en microorganismes lorsque les conditions de dilution ou de mortalité sont insuffisantes sur certaines zones sensibles, de type conchylicole ou balnéaire. A côté de procédés fiables, si ils sont bien gérés, comme l'infiltration ou le lagunage, il existe des filières de désinfection chimique qui exigent, pour être efficaces et les moins polluantes possibles, d'être employées sur des effluents parfaitement épurés d'un point de vue chimique (faibles teneurs en matières en suspension, en matière organique et en ammoniaque); néanmoins, l'hypothèse de phé- 
nomènes de reviviscence des germes après traitement ne peut pas être écartée et le contrôle de l'efficacité de la désinfection chimique repose sur l'utilisation des germes-test témoins de contaminations fécales qui sont souvent moins résistants aux traitements que certaines bactéries et virus pathogènes; c'est pourquoi des recherches se poursuivent actuellement pour la mise au point de nouvelles filières de désinfection des eaux usées utilisant l'acide péracétique, l'ozone, les U.V., l'irradiation par faisceau d'électrons ou la micro-filtration (Audic, 1991).

\section{BIBLIOGRAPHIE}

Aminot A. \& Guillaud J.F., 1991. Apports en matière organique et en sels nutritifs par les stations d'épuration. IFREMER, Actes de Colloques 11 "La mer et les rejets urbains". Bendor 13-15 juin 1990: 11-26.

Aminot A., Guillaud J.F. \& Le Guellec A.M., 1990. Suivi à long terme des apports de matières organiques et nutritives par les effluents urbains de Morlaix. IFREMER DRO.EL 90-30: $42 \mathrm{p}$.

Audic J.M., 1991. Evolution des technologies d'élimination des microorganismes. IFREMER, Actes de Colloques 11 "La mer et les rejets urbains ". Bendor 13-15 juin 1990: 133148.

Dion P., 1988. Etude de certains aspects écophysiologiques des marées vertes. Rapport de constat CEVA Pleubian/IFREMER : $37 \mathrm{p}$.

Dupray E., Baleux B., Bonnefond J.L., Guichaoua C., Pommepuy M. \& Derrien A., 1991. Apports en bactéries par les stations d'épuration. IFREMER Actes de Colloques 11 "La mer et les rejets urbains ". Bendor 13-15 juin 1990: 81-88.

Giovanelli G., Bonasoni P., Loglio G., Ricci C., Tesci U. \& Cini R., 1988. Evidence of anionic-surfactant enrichment in marine aerosol. Marine Poll. Bull. 19 (6) : 274-277.

Guillaud J.F., 1987. Study program on the discharge of urban wates into the sea. Pollution and the coastal environment: a symposium. Maryland Sea Grand Publication. College Park MD, USA: 52-57.

Guillaud J.F. \& Aminot A., 1991. Devenir des éléments nutritifs en zone littorale. IFREMER, Actes de Colloques 11 "La mer et les rejets urbains ". Bendor 1315 juin 1990: 27-34.

Lassus P., Bogé G., Gentien P., Loarer R., Pagano G. \& Quiniou F., 1991. Toxicité des rejets urbains. IFREMER, Actes de Colloques 11 "La mer et les rejets urbains". Bendor 13-15 juin 1990: 171-186.

Le Hir P., Guillaud J.F., Bassoullet Ph. \& L'Yavanc J., 1991. Application d'un modèle sédimentaire au devenir des contaminants particulaires. IFREMER, Actes de Colloques 11 "La mer et les rejets urbains". Bendor 13-15 juin 1990: 205-220.

Lerat Y., 1990. Cycles annuels de la matière organique et des éléments nutritifs dans les sédiments d'un écosystème côtier (baie de Morlaix, France). Interaction avec le comportement pélagique. Thèse Univ. de Bret. Occ., Brest: $212 \mathrm{p}$.

Marchand M., 1985. Processus géochimiques d'apports et de distribution de polluants organiques dans l'environnement marin. Thèse Univ. de Paris VI: $309 \mathrm{p}$

Marchand M., Caprais J.C., Pignet P. \& Porot V., 1989. Les polluants organiques dans les effluents urbains et apports polluants dans le milieu marin. Cas du littoral français. Water Res., 23 (4) : 461-470. 
Martin G., 1979. Le problème de l'azote dans les eaux. Tech. et Doc. Paris. $279 p$.

Ménesguen A., 1991. Présentation du phénomène d'eutrophisation littorale. IFREMER, Actes de Colloques 11 "La mer et les rejets urbains ". Bendor 1315 juin 1990: 35-52.

Ministère de l'Environnement, 1983. Assainissement en zone littorale. Cahiers Techniques de la Direction de la Prévention des Pollutions $n^{\circ} 11$ : $92 p$.

Queguiner B., 1988. Synthèse opérationnelle du programme pluriannuel baie de Quiberon-Vilaine. Rapport de l'Univ. Bret. Occidentale, Brest, $34 \mathrm{p}$.

Pommepuy M., Guillaud J.F., Martin Y., Dupray E., L'Yavanc J., Derrien A. \& Cormier M., 1991. Le devenir des bactéries en zone littorale. IFREMER, Actes de Colloques 11 "La mer et les rejets urbains". Bendor 13-15 juin 1990: 89-100.

Prieur D., Mevel G., Nicolas J.L., Plusquellec A. \& Vigneulle M., 1990. Interactions between bivalve molluscs and bacteria in the marine environment. Oceanogr. mar. Biol. Annu. Rev., 28: 277-352.
Romana L.A., Boutier B., Marchand M., Loarer R., Brisset P., Caillot A. \& Arnoud A., 1991. Apports en micropolluants organiques et minéraux. IFREMER Actes de Colloques 11 "La mer et les rejets urbains". Bendor 1315 juin 1990: 153-170.

Ryther J.H. \& Dunstan W.M., 1971. Nitrogen, phosphorus and eutrophication in the coastal marine environment. Science (171): 1008-1013.

Salomon J.C., Breton M. \& Pommepuy M., 1991. Intérêt d'un modèle de transport dissous pour les rejets urbains en zone estuarienne. IFREMER, Actes de Colloques 11 "La mer et les rejets urbains". Bendor 13-15 juin 1990: 191204.

Schaack F., Bosquet A.F., Chevalier D., Kerlan F. \& Senelier Y., 1985. Efficacité des stations d'épuration biologiques actuelles à l'égard de la pollution phosphatée. T.S.M. L'eau (80) 4 : 173181.

Thouvenin B., 1991. Caractéristiques et modélisation d'un rejet par émissaire. IFREMER Actes de Colloques 11 "La mer et les rejets urbains ". Bendor 1315 juin 1990: 221-236. 